\title{
Contributions of Nrf2 to Puerarin Prevention of Cardiac Hypertrophy and its Metabolic Enzymes Expression in Rats ${ }^{[\text {[ }}$
}

\author{
Gan-Jian Zhao, ${ }^{1}$ Ning Hou, ${ }^{1}$ Shao-Ai Cai, ${ }^{1}$ Xia-Wen Liu, ${ }^{1}$ Ai-Qun Li, Chuan-Fang Cheng, \\ Yin Huang, Li-Rong Li, Yun-Pei Mai, Shi-Ming Liu, Cai-Wen Ou, Zhen-Yu Xiong, \\ Xiao-Hui Chen, Min-Sheng Chen, and Cheng-Feng Luo
}

The Second Affiliated Hospital of Guangzhou Medical University, Guangzhou Institute of Cardiovascular Disease (G.-J.Z., A.-Q.L., C.-F.C., Y.H., L.-R.L., S.-M.L., C.-F.L.), School of Pharmaceutical Sciences and the Fifth Affiliated Hospital, Guangzhou Medical University (N.H., X.-W.L., Y.-P.M.), The Second Affiliated Hospital of Guangzhou Medical University (S.-A.C., X.-H.C.); Zhujiang Hospital, Southern Medical University, Guangdong Provincial Center of Biomedical Engineering for Cardiovascular Disease (C.-W.O., M.-S.C.), and The First Affiliated Hospital, Sun Yat-sen University (Z.-Y.X.), Guangzhou, China

Received February 9, 2018; accepted May 21, 2018

\section{ABSTRACT}

Previous evidence has suggested that puerarin may attenuate cardiac hypertrophy; however, the potential mechanisms have not been determined. Moreover, the use of puerarin is limited by severe adverse events, including intravascular hemolysis. This study used a rat model of abdominal aortic constriction (AAC)-induced cardiac hypertrophy to evaluate the potential mechanisms underlying the attenuating efficacy of puerarin on cardiac hypertrophy, as well as the metabolic mechanisms of puerarin involved. We confirmed that puerarin (50 mg/kg per day) significantly attenuated cardiac hypertrophy, upregulated Nrf2, and decreased Keap1 in the myocardium. Moreover, puerarin significantly promoted Nrf2 nuclear accumulation in parallel with the upregulated downstream proteins, including heme oxygenase 1, glutathione transferase $\mathrm{P1}$, and $\mathrm{NAD}(\mathrm{P}) \mathrm{H}$ :quinone oxidoreductase 1 . Similar results were obtained in neonatal rat cardiomyocytes (NRCMs) treated with angiotensin II (Ang II; $1 \mu \mathrm{M})$ and puerarin (100 $\mu \mathrm{M})$, whereas the silencing of Nrf2 abolished the antihypertrophic effects of puerarin. The mRNA and protein levels of UGT1A1 and UGT1A9, enzymes for puerarin metabolism, were significantly increased in the liver and heart tissues of AAC rats and Ang II-treated NRCMs. Interestingly, the silencing of Nrf2 attenuated the puerarin-induced upregulation of UGT1A1 and UGT1A9. The results of chromatin immunoprecipitationquantitative polymerase chain reaction indicated that the binding of Nrf2 to the promoter region of Ugt1a1 or Ugt1a9 was significantly enhanced in puerarin-treated cardiomyocytes. These results suggest that Nrf2 is the key regulator of antihypertrophic effects and upregulation of the metabolic enzymes UGT1A1 and UGT1A9 of puerarin. The autoregulatory circuits between puerarin and Nrf2induced UGT1A1/1A9 are beneficial to attenuate adverse effects and maintain the pharmacologic effects of puerarin.

\section{Introduction}

Puerarin (7, $4^{\prime}$-dihydroxyisoflavone- $8 \beta$-glucopyranoside) is a major active ingredient in the Chinese medicine Pueraria radix, which is extracted from the kudzu root [Pueraria lobota (Wild) Howe]. The pharmacologic benefits of puerarin include improvement of microcirculation, scavenging of oxygen free radicals, and amelioration of insulin resistance (Zhou et al.,

This work was supported by the National Natural Science Foundation of China [Grants 81374009, U1501222, 81402928], Guangdong Natural Science Foundation [Grant 2014A030313493], Guangzhou Education Bureau [Grant 2012C090], and The Key Medical Disciplines and Specialties Program of Guangzhou [2017-2019].

${ }^{1}$ G.-J. Z., N. H., S.-A. C. and X.-W. L. contributed equally to this work.

https://doi.org/10.1124/jpet.118.248369.

S This article has supplemental material available at jpet.aspetjournals.org. 2014b), which make it a potential treatment of patients with hypertension (Song et al., 1988), cerebral ischemia (Gao et al., 2009), myocardial ischemia (Zhang et al., 2006), diabetes mellitus (Hsu et al., 2003), and arteriosclerosis (Yan et al., 2006).

Physiologically, cardiac tissue exhibits plasticity that enables the heart to respond to various physiologic or pathologic stresses. Under the continuous stress induced by pathologic conditions, including hypertension and aortic stenosis, pathologic hypertrophy is characterized by ventricular remodeling, with the impairment of cardiac systolic and diastolic function, which eventually lead to deleterious outcomes, such as heart failure, arrhythmia, and sudden cardiac death (Oka et al., 2014; Kamo et al., 2015). Therefore, preventative strategies against the incidence of pathologic cardiac hypertrophy may

ABBREVIATIONS: AAC, abdominal aortic constriction; AhR, aryl hydrocarbon receptor; Ang II, angiotensin II; ANP, atrial natriuretic peptide; ARE, antioxidant/electrophile-response element; CAR, constitutive androstane receptor; cDNA, complementary DNA; ChIP, Chromatin immunoprecipitation; GSTP1, glutathione transferase p 1; HO1, heme oxygenase 1; IVSd, interventricular septum diameter; LVEDP, left ventricular end-diastolic pressure; LVEF, left ventricular ejection fraction; LVESP, left ventricular end-systolic pressure; LVFS, left ventricular fractional shortening; LVId, left ventricular interior diameter; LVPWd, Left ventricular posterior wall diameter; NQO1, NAD(P)H:quinone oxidoreductase 1; NRCMs, neonatal rat cardiomyocytes; Nrf2, nuclear factor erythroid 2-related factor 2; RT-PCR, reverse transcriptionpolymerase chain reaction; PSR, picrosirius red staining; PXR, pregnane $X$ receptor; UGTs, UDP-glucuronosyltransferases. 
be important to improve the outcome in patients at risk of developing heart failure. Puerarin has been suggested to confer antihypertrophic efficacy in the heart (Yuan et al., 2014; Liu et al., 2015). Results of our previous studies showed that puerarin may prevent cardiac hypertrophy via the restoration of the AMPK/mTOR-mediated autophagy pathway (Liu et al., 2015). Moreover, other studies have shown that puerarin may exert antihypertrophic efficacy via other mechanisms, such as the blockade of hypertrophy-related signaling pathways (Chen et al., 2014; Yuan et al., 2014), inhibition of oxidative stress (Gang et al., 2015; Hou et al., 2017), and enhancement of miR-15b and miR-195 expression (Zhang et al., 2016); however, the key regulator involved in the potential antihypertrophic efficacy of puerarin remains to be determined.

Understanding the metabolic pathways of puerarin is important to optimize its pharmacologic efficacy and to limit its potential adverse effects. Puerarin has a very low water solubility, with a limited absolute bioavailability of $1.8 \%$ after oral administration (Luo et al., 2011a,b; Li et al., 2014). Generally, xenobiotics experience absorption, metabolism, disposition, and excretion after administration. Puerarin-7-O-glucuronide is one of the major metabolites of puerarin in rats (Luo et al., $2012,2013)$. Our previous studies confirmed that seven UDP-glucuronosyltransferase (UGT) isoforms (UGT1A1, 1A9, $1 \mathrm{~A} 10,1 \mathrm{~A} 3,1 \mathrm{~A} 6,1 \mathrm{~A} 7$, and $1 \mathrm{~A} 8)$ can catalyze the formation of its major metabolite, puerarin-7-O-glucuronide, and UGT1A1 is the primary enzyme responsible for the metabolism of puerarin (Luo et al., 2012). Moreover, transcription factors, such as constitutive androstane receptor $(\mathrm{CAR})$, pregnane $\mathrm{X}$ receptor (PXR), and aryl hydrocarbon receptor (AhR), can enhance the transcriptions of UGT isoforms, including UGT1A1 (Sugatani et al., 2005; Mackenzie et al., 2010); however, recent evidence suggests that the transcription factor nuclear factor erythroid 2-related factor 2 (Nrf2) plays a key role in preserving cardiac function, particularly via the regulation of cardiac hypertrophy, evidenced by the enhanced cardiac hypertrophy and diastolic dysfunction in Nrf2 knockout mice (Erkens et al., 2015). Therefore, we hypothesized that puerarin may prevent cardiac hypertrophy by enhancing the activity of Nrf2. Thus, the aim of the current study was to investigate whether the potential antihypertrophic efficacy of puerarin is associated with the regulatory effect of Nrf2 in a pressure-overload rat model and angiotensin II (Ang II)-treated neonatal rat cardiomyocytes. Moreover, we aimed to evaluate whether puerarin may also induce UGT1A1 and 1A9 in liver and heart tissue via the regulation of Nrf2. Our results showed that Nrf2 is a common transcription factor in the effects of puerarin and that it protects against cardiac hypertrophy and regulates puerarin-metabolizing enzymes.

\section{Materials and Methods}

Chemicals. Injectable puerarin was purchased from Zhejiang Zhenyuan Pharmaceutical Co., Ltd. (Shaoxing, China). Puerarin and Ang II were purchased from Sigma Chemical (St. Louis, MO). SuperSignal West Pico Chemiluminescent Substrate was obtained from Thermo (Rockford, IL). TRIzol reagent was purchased from Ambion Inc. (Life Technologies Inc., Grand Island, NY). A First-Strand cDNA Synthesis kit was purchased from Takara Bio Inc. (Shiga, Japan). The Coomassie Protein Assay kit was purchased from Bio-Rad Laboratories (Hercules, CA).
Animal Model. Half of the male and female pathogen-free SpragueDawley rats (150-180 g) were purchased from Guangdong Medical Laboratory Animal Center (Guangzhou, China). All animal experiments were performed according to the EC Directive 86/609/EEC. The protocol of the animal study was reviewed and approved by the ethics committee of Guangzhou Medical University before performance. The rats were housed in an environmentally controlled room (temperature, $25 \pm 2{ }^{\circ} \mathrm{C}$; humidity, $60 \% \pm 5 \%$; 12-hour dark/light cycle) with access to regular chow diet ad libitum for 1 week before the experiments. Cardiac hypertrophy was induced by abdominal aortic constriction (AAC), which was modified based on a previously reported procedure (Reddy et al., 1996; Huang et al., 2015; Ku et al., 2016). In brief, the rats were anesthetized using pentobarbital ( $45 \mathrm{mg} / \mathrm{kg}$, i.p. injection). The abdominal aorta was then exposed above the kidneys and constricted at the suprarenal level by a 7-0 silk suture tied around both the aorta and a blunted 18-gauge needle, which was subsequently removed. A similar procedure without abdominal arterial ligation was performed as a sham surgery. On completion of surgery, the rats were injected with buprenorphine and placed down on a warming pad until they woke. Baseline echocardiography assessment was performed 1 week after the surgeries, and the rats were randomly divided into four groups. The sham group included sham rats that received daily intraperitoneal injection of vehicle, the AAC group included AAC rats with vehicle injection daily, the AAC-Pue group included AAC rats with an injection of puerarin ( $50 \mathrm{mg} / \mathrm{kg}$ body weight) daily, and the Pue group included sham rats with an injection of puerarin $(50 \mathrm{mg} / \mathrm{kg}$ body weight) daily. Each group contained four male and four female rats. Echocardiography assessments were performed every 2 weeks for the rats in each group. After 6 weeks of puerarin administration, the rats were subjected to catheter-based hemodynamic assessment, and they were sacrificed after hemodynamic assessment. Their heart and liver tissues were extracted and divided for mRNA, protein, histology, and immunofluorescence analysis.

Echocardiography. Cardiac function was evaluated by transthoracic echocardiography with a $250-\mathrm{MHz}$ ultrasound transducer (Vevo 2100; VisualSonics, Toronto, ONT). The rats were sedated using $2 \%$ isoflurane and maintained with $1 \%-1.5 \%$ isoflurane. The parasternal short-axis view (B-mode, M-mode) was obtained, and cardiac structure and function were measured. Left ventricular posterior wall diameter (LVPWd), left ventricular interior diameter (LVId), and interventricular septum diameter (IVSd) were measured from the LV M-mode tracing at the midpapillary muscle level, and parameters of the left ventricular ejection fraction (LVEF) and fractional shortening (LVFS) were calculated.

Noninvasive Measurement of Blood Pressure and CatheterBased Analysis of Hemodynamic Parameters. Rat tails were fixed gently and then subjected to a CODA monitor (Kent Scientific, Torrington, CT). Noninvasive blood pressure testing was done at least five times for each animal. The mean systolic pressure and diastolic pressure were recorded. For catheter-based hemodynamic measurements, after the rats were anesthetized with $30 \%$ urethane, a catheter transducer was inserted via the right carotid artery into the left ventricle. The parameters left ventricular end-systolic pressure (LVESP), left ventricular end-diastolic pressure (LVEDP), maximal rate of pressure development $(\mathrm{Max}+\mathrm{dP} / \mathrm{dt}$ ), and maximal rate of pressure decay (Max - dp/dt) were analyzed using the PowerLab system (ADInstruments, Sydney, Australia) and recorded.

Histology and Immunohistochemical Staining. The rats were weighed and euthanized using cervical dislocation. Hearts were removed and perfused briefly with $10 \% \mathrm{KCl}$ to arrest the heart in diastole. The hearts were then fixed with $10 \%$ formalin and integrated into paraffin. For histologic evaluations with H\&E, Masson's trichrome, and picrosirius red (PSR) staining, tissues from four rats of each group were embedded with paraffin and cut into $4-\mu \mathrm{m}$ sections as previously described (Yuan et al., 2014). For immunohistochemical staining, antigen retrieval was performed in EDTA buffer ( $\mathrm{pH} 9.0)$ by heating to $99^{\circ} \mathrm{C}$ for 20 minutes. After quenching endogenous peroxidase with $3 \% \mathrm{H}_{2} \mathrm{O}_{2}$, the sections were blocked with $10 \%$ 
nonimmune goat serum (Life Technologies) and then incubated with primary antibodies, including NRF2, KEAP1, UGT1A1, or UGT1A9, as shown in Supplemental Table S1 at $4^{\circ} \mathrm{C}$ overnight. Signals were amplified with the Histostain-SP Kit (Life Technologies) and detected with DAB substrate (Dako North America, Inc., Carpinteria, CA). Hematoxylin was used as a counterstain. The images were visualized and captured under a microscope (Eclipse TS 100; Nikon, Tokyo, Japan).

Isolation, Culture, and Treatment of Neonatal Rat Cardiomyocytes. The neonatal rat cardiomyocytes (NRCMs) were isolated from neonatal Sprague Dawley rats (1 to 2 days old) with the assistance of the Neonatal Cardiomyocyte Isolation System purchased from Worthington (Lakewood, NJ) based on the Simpson method (Simpson and Savion, 1982) as previously reported (Hou et al., 2017). The isolated NRCMs were seeded with a density of $5 \times 10^{6}$ cells $/ \mathrm{ml}$ in Dulbecco's modified Eagle's medium containing $10 \%$ fetal bovine serum and $0.1 \mathrm{mM}$ bromodeoxyuridine. The NRCMs were then treated with vehicle alone, Ang II $(1 \mu \mathrm{M})$ alone, Ang II $(1 \mu \mathrm{M}) /$ puerarin $(100 \mu \mathrm{M})$, or puerarin alone $(100 \mu \mathrm{M})$ for 24 hours before the cells were harvested for analysis.

Nrf2 siRNA Transfection. For Nrf2-siRNA transfection, the cardiomyocytes were transfected with Nrf2-siRNA or Nrf2-negative control siRNA (Supplemental Table S2 (Viewsolid Biotech, Beijing, China) for 48 hours upon plating the cells in a six-well plate or 60 -mm dish. The transfected cells were treated with Ang II $(1 \mu \mathrm{M})$ alone, Ang II $(1 \mu \mathrm{M}) /$ puerarin $(100 \mu \mathrm{M})$, or puerarin alone $(100 \mu \mathrm{M})$ for 24 hours, followed by further analysis.

Cell Surface Area Measurement. The images of treated cardiomyocytes were obtained using a high-power microscope (Eclipse TS 100; Nikon). The surface area was determined with NIH Image J software. Results were calculated based on 100 cells randomly selected from five wells for each group.

RNA Isolation, Quantitative RT-PCR. Total RNA was isolated using TRIzol reagent (Life Technologies) from the left ventricular tissue or treated cardiomyocytes. Complementary DNA (cDNA) was synthesized using the First-Strand cDNA Synthesis kit (Life Technologies) according to the manufacturer's instructions. Real-time PCR was performed using a QuantiTect SYBR Green PCR Kit (Takara Bio Inc.) on an ABI StepOneTM real-time PCR system (Thermo Fisher Scientific Inc., Waltham, MA). The sequences of the primer pairs for each gene are shown in Supplemental Table S2. GAPDH was used as normalization for qPCR.

Western Blot Analysis. Whole-protein extraction was prepared from left ventricular tissue or treated cardiomyocytes using RIPA buffer with protease and phosphatase inhibitors (BestBio Co., Shanghai, China). The proteins from the nucleus and cytoplasm were prepared using the NE-PER nuclear and cytoplasmic extraction kit (Thermo Fisher Scientific) following the manufacturer's protocol. Protein concentrations were quantified using the BCA kit (Thermo Fisher Scientific). Equal protein amounts were resolved on $10 \%$ sodium dodecyl sulfate polyacrylamide gel electrophoresis and transferred to polyvinylidene difluoride membranes. The membrane was blocked with $5 \%$ no-fat milk in Tris-buffered saline $0.1 \%$ Tween-20 for 1 hour and then incubated with primary antibodies of interest (all antibodies are shown in Supplemental Table S1) at $4^{\circ} \mathrm{C}$ overnight. The blot was then incubated with peroxidase-conjugated secondary antibody (ImmunoWay Biotechnology Co., Plano, TX) for 1 hour at room temperature. Bands of the proteins were observed using SuperSignal West Pico Chemiluminescent substrate (Thermo), and quantified analyses were performed with densitometry using NIH Image J software.

Immunofluorescence of Cardiomyocytes. The treated cardiomyocytes were fixed with $4 \%$ paraformaldehyde for 10 minutes and permeabilized with $0.05 \%$ Triton X-100 for 15 minutes. The nonspecific binding sites were blocked for 1 hour with $5 \%$ bovine serum albumin in phosphate-buffered saline. The cells were then incubated with NRF2 antibody (1:200) overnight, followed by immunostaining with Dylight 488 anti-rabbit antibody (Thermo Fisher Scientific) for 1 hour at room temperature. Nuclear counterstaining was performed with
4', 6-diamidino-2-phenylindole (DAPI; Sigma-Aldrich). Coverslips were mounted on slides, and images were captured with a fluorescence microscope (Eclipse Ni-u; Nikon) using NIS-Elements software.

Chromatin Immunoprecipitation Assay. A ChIP assay was performed with a ChIP assay kit (EZ-ChIP, Catalog no. 17-371; Thermo Fisher Scientific) according to the manufacturer's instruction. Briefly, cardiomyocytes were incubated with $1 \%$ formaldehyde to crosslink the proteins to the DNA. Sonication was performed to shear the chromatin to a 200-1000 bp of DNA. The size of the DNA was verified by agarose gel electrophoresis. For the immunoprecipitation step, the primary antibody of NRF2 (1:100, CST) was used, followed by protein $\mathrm{G}$-conjugated agarose beads to mutually enrich the protein and DNA complexes. Immunoprecipitated chromatins were incubated at $65^{\circ} \mathrm{C}$ in $5 \mathrm{M} \mathrm{NaCl}$ overnight to reverse cross-links. After digestion with proteinase K, DNA was recovered by phenol/chloroform extraction and ethanol precipitation. Precipitated DNA was analyzed by real-time PCR with the primers listed in Supplemental Table S2.

Statistical Analysis. Data were presented as the means \pm S.E. Statistical analysis was performed with SPSS 18.0 software (IBM Corporation, Armonk, NY). The statistical differences between the groups were analyzed by one-way analysis of variance followed by a post hoc Tukey test. $P<0.05$ was statistically significant.

\section{Results}

Puerarin Attenuated AAC-Induced Cardiac Hypertrophy. Using a rat model of AAC-induced cardiac hypertrophy, we evaluated whether puerarin may attenuate cardiac hypertrophy and ventricular remodeling at a dose of $50 \mathrm{mg} / \mathrm{kg}$ per day administered from 1 week after the surgery. Approximately $10 \%$ of the rats died within 24 hours after surgery; however, no more deaths were detected during the subsequent 7 weeks of the experiment, which indicates at least eight rats for each group at the end of the study. Increased cardiac mass (Fig. 1A), myocyte cross sectional area (Fig. 1B), heart weight/body weight (HW/BW) ratio, and heart weight/tibial length (HW/TL) ratio (Fig. 1E) were observed in the rats subjected to AAC surgery after 7 weeks compared with the sham group, suggesting the exhibition of cardiac hypertrophy in the AAC group. Interestingly, injection with puerarin for 6 weeks significantly attenuated the above hypertrophic manifestations (Fig. 1, A, B, and E). We further examined the expression of hypertrophic genes. As shown in Fig. $1 \mathrm{~F}$, the mRNA expression of atrial natriuretic peptide (Anp) and $\beta$-myosin heavy chain ( $M y h 7)$, which were upregulated after ACC surgery, were significantly blunted in the puerarin injection rats. A key feature of cardiac hypertrophy and remodeling is myocardial fibrosis. Therefore, Masson trichrome and PSR staining on cardiac tissue sections were performed to evaluate the severity of myocardial interstitial and perivascular fibrosis in each group. As shown in Fig. 1, C and D, interstitial and perivascular fibrosis were remarkable after 7 weeks of AAC, which were ameliorated by puerarin administration. The protective effect of puerarin on cardiac hypertrophy induced by AAC was evaluated by echocardiograph. As shown in Fig. 2, 7 weeks after ACC surgery, hypertrophic indicators such as LVPWd and IVSd increased significantly in the AAC group compared with the sham group (Fig. 2, A-C). Conversely, LVId decreased (Fig. 2D) in rats with AAC surgery. Puerarin administration resulted in a significant reversal of these hypertrophic indicators (Fig. 2, A-D). The LVEF and LVFS did not show significant changes in each group at the end of this study (Fig. 2E). 
A

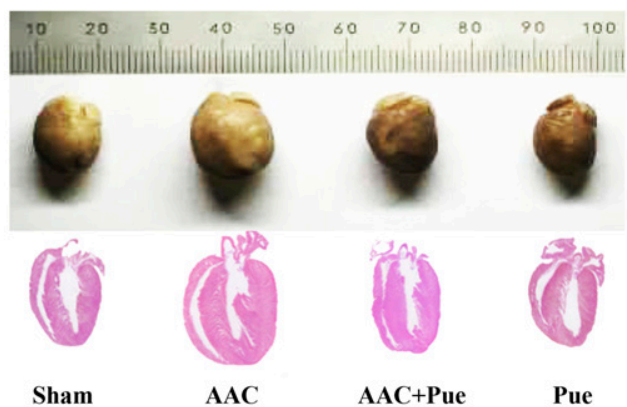

C

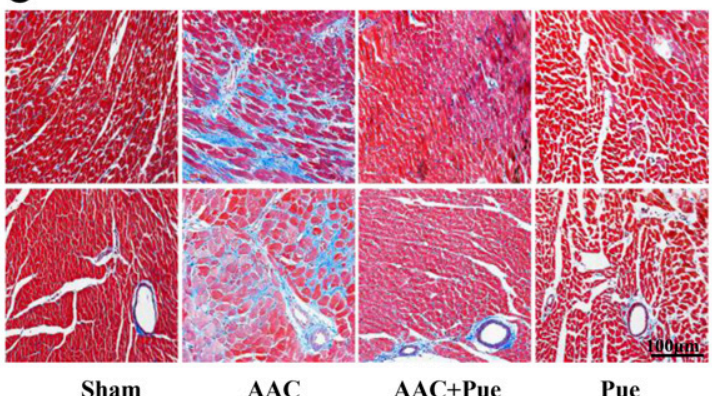

Sham

AAC + Pue

Pue

B

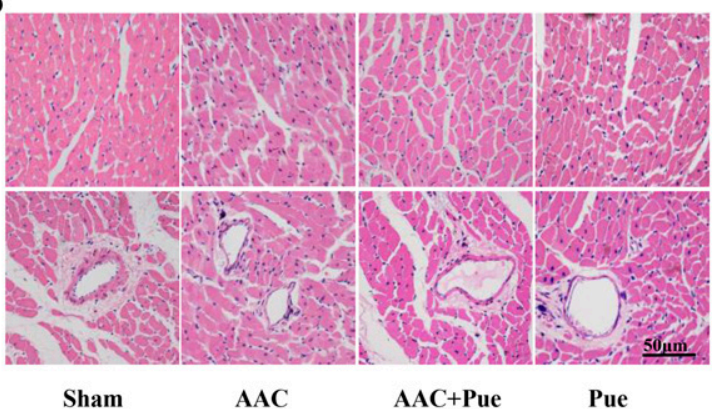

D

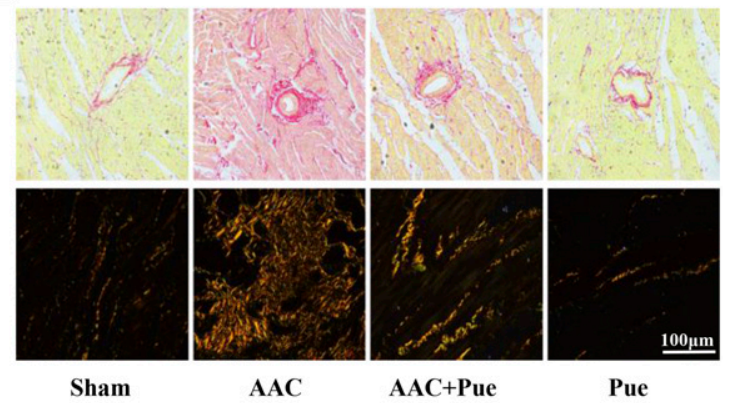

$\mathrm{HW} / \mathrm{BW}$
$\mathrm{HW} / \mathrm{TL}$

E

F

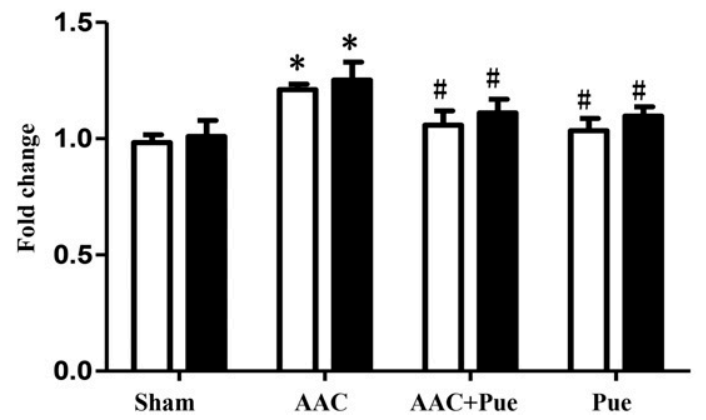

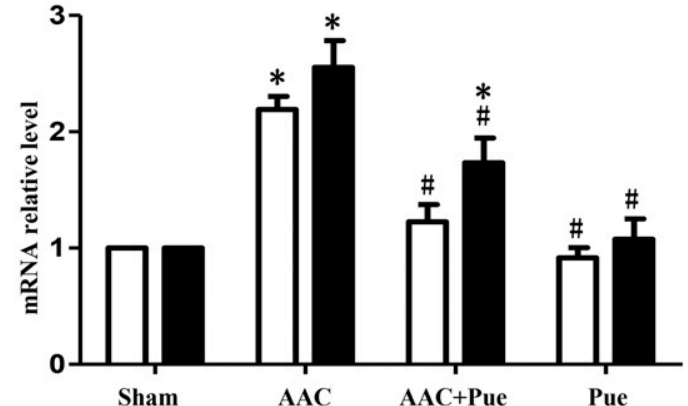

Fig. 1. Puerarin protected against AAC-induced cardiac hypertrophy. (A) Gross hearts (upper); H\&E staining for longitudinal section of rat heart (lower). (B) H\&E staining of sham and AAC hearts after 6 weeks of puerarin treatment. Cross-section of myocardium (upper). Perivascular cardiomyocytes (lower). (C) Representative images of Masson trichrome staining for interstitial fibrosis of myocardium (upper) and perivascular fibrosis (lower). (D) Picrosirius red staining indicated myocardial fibrosis representatively by upright microscopy (upper) and polarization microscope (lower). (E) Heart weight/body weight (HW/BW) ratio and heart weight/tibial length (HW/TL) ratio. (F) RT-PCR analysis for atrial natriuretic peptide (Anp) and $\beta$-myosin heavy chain $(M y h 7)$ mRNA expression. AAC + Pue, puerarin-injected AAC group; Pue, puerarin injected-sham group. Representative images from three sets of staining are shown. ${ }^{*} P<0.05$ vs. sham group; ${ }^{\#} P<0.05$ vs. AAC group, $n=8$ for each group. Each group contained equal numbers of male and female rats.

Puerarin Improved AAC-Induced Hemodynamic Parameters. Catheter-based hemodynamic measurements were performed at the end of the study to further detect the changes of left ventricular systolic and diastolic function. As shown in Table 1, 7 weeks of AAC resulted in significantly increased systolic blood pressure, diastolic blood pressure, and LVESP. Puerarin administration normalized these hemodynamic parameters; however, the parameters, including LVEDP, Max $+\mathrm{dP} / \mathrm{dt}$, and Max $-\mathrm{dp} / \mathrm{dt}$ did not differ significantly between the groups.

Puerarin Protected against Cardiac Hypertrophy and Oxidative Stress via Activation of Nrf2 In Vivo. Nrf2 is the master regulator of the antioxidant response. Kelch-like
ECH-associated protein 1 (Keap1) is an E3 ubiquitin ligase substrate adaptor that regulates the level of Nrf2 protein in a redox-dependent fashion. Stress-related modification of Keap1 results in Nrf2 stabilization, accumulation of the transcription factor in the nucleus, where Nrf2 binds to the antioxidant/ electrophile-response element (ARE), and protects against oxidative stress damage (Rada et al., 2011). To assess whether puerarin can activate the Nrf2-Keap1 pathway in rats with AAC surgery, we evaluated the transcription of Nrf2 mRNA by RT-PCR and the protein expression of NRF2 by Western blotting in the liver and heart tissues from each group (Fig. 3). Although NRF2 protein expression did not show a significant difference between the sham group and AAC group in rat livers, its mRNA 
A

A
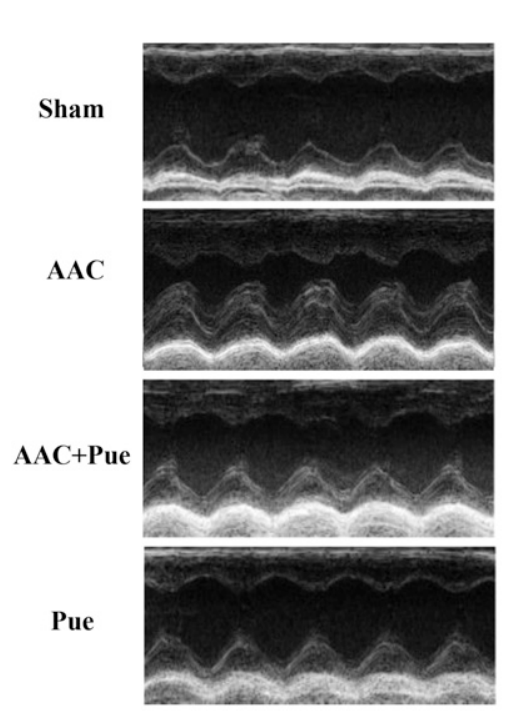

B
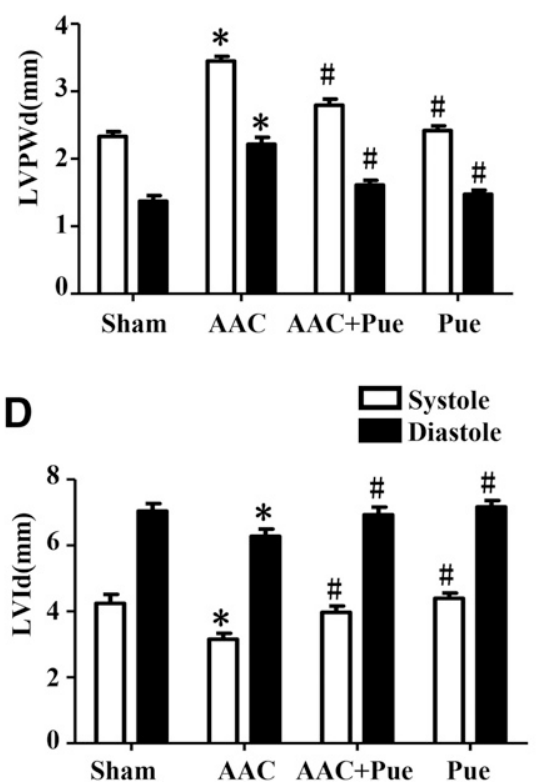

C

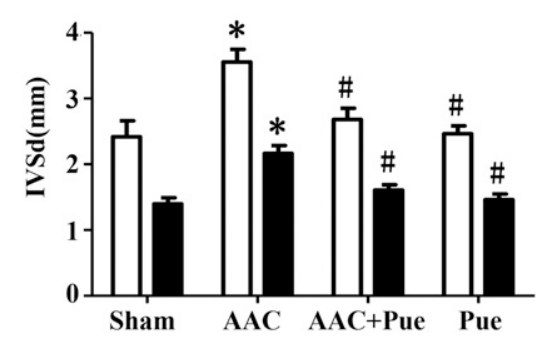

E

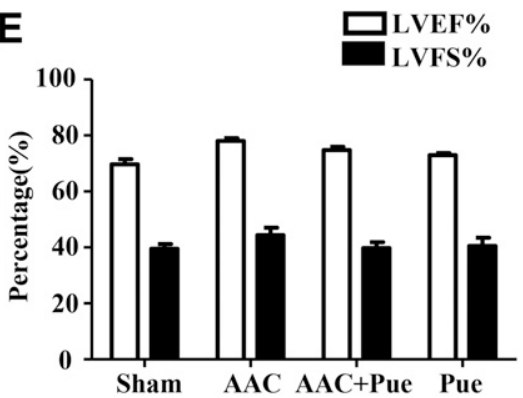

Fig. 2. Puerarin improved AAC-induced cardiac hypertrophy as indicated by echocardiographic examinations. (A) Representative M-mode images of the indicated groups. (B-D) Parameters of cardiac structure: IVSd, LVId, LVPWd. (E) LVEF and LVFS. ${ }^{*} P<0.05$ vs. sham group; $P<0.05$ vs. AAC group, $n=8$ for each group (four male and four female rats).

and protein levels were significantly decreased in AAC hearts compared with the control. Moreover, puerarin treatment significantly increased the Nrf2 mRNA and protein levels in the hearts and livers from the AAC and sham rats, accompanied by decreased KEAP1 protein (Fig. 3, A-D). Because NRF2 accumulation in the nucleus may indicate its activation, we further detected its protein expression in the nuclear and cytoplasmic fractions of heart lysates. As shown in Fig. 3E, compared with the sham group, rats from the AAC surgery groups exhibited significantly decreased translocation of NRF2 from the cytosol to the nuclear fraction. In contrast, puerarin significantly enhanced its nucleus accumulation. The cytosolic NRF2 level was not significant different between the groups. Immunohistochemical staining also showed an increment in nuclear NRF2 but a reduction in KEAP1 in AAC-Pue rats compared with AAC rats (Fig. 3, G and $\mathrm{H}$ ). We further measured the protein levels of heme oxygenase 1 (HO1), glutathione transferase 1 (GSTP1), and NAD(P)H:quinone oxidoreductase 1 (NQO1), which are well known downstream antioxidant proteins of Nrf2. The expression of HO1, GSTP1, and NQO1 was decreased in rats with AAC surgery compared with those in the sham group. The difference in GSTP1 and NQO1 were statistically significant; however, puerarin remarkably increased their protein levels in both AAC-Pue and Pue rats (Fig. 3F).

Puerarin Induced Nrf2 Nuclear Accumulation in AngII-Induced Cardiomyocytes Hypertrophy. To determine more fully whether puerarin could activate Nrf2 in the pathophysiologic process of cardiac hypertrophy, we examined its effects on hypertrophic changes in NRCMs resulting from Ang II. Treatment with Ang II $(1 \mu \mathrm{M})$ resulted in a significantly increased cell- surface area $(P<0.05$; Fig. $4, \mathrm{~A}$ and B) and upregulated the mRNA levels of Anp and Myh7 (Fig. 4C). More importantly, these hypertrophic changes were attenuated by coincubation with puerarin (100 $\mu \mathrm{M}$, Fig. $4, \mathrm{~A}-\mathrm{C})$. We further evaluated the NRF2 and KEAP1 protein levels using Western blotting. As shown in Fig. 4D, the NRF2 protein levels were significantly higher in the overall and nuclear lysates from cardiomyocytes treated with puerarin alone than control cells. Reduced NRF2 and increased KEAP1

TABLE 1

Hemodynamic parameters in rats without or with puerarin administered 6 weeks postsurgery Systolic blood pressure (SBP) and diastolic blood pressure (DBP) detected by rat tail artery blood pressure mesurement.

\begin{tabular}{lcccc}
\hline & Sham & AAC & AAC+Pue & Pue \\
\hline SBP (mm Hg) & $122.8 \pm 5.8$ & $149.5 \pm 13.9^{*}$ & $132.9 \pm 11.1^{* *}$ & $124.1 \pm 3.9^{* *}$ \\
DBP (mm Hg) & $95.6 \pm 7.9$ & $120.1 \pm 12.9^{*}$ & $98.7 \pm 10.5^{* *}$ & $96.4 \pm 5.3^{* *}$ \\
LVESP (mm Hg) & $114.8 \pm 13.5$ & $144.2 \pm 10.4^{*}$ & $121.2 \pm 14.8^{* *}$ & $116.6 \pm 18.9^{* *}$ \\
LVEDP (mm Hg) & $5.1 \pm 2.6$ & $5.5 \pm 3.2$ & $5.3 \pm 2.9$ & $5.3 \pm 2.9$ \\
Max +dp/dt (mm Hg/s) & $4078 \pm 587$ & $4289 \pm 1141$ & $3835 \pm 884$ & $3881 \pm 497$ \\
Max -dp/dt (mm Hg/s) & $-3667 \pm 629$ & $-3471 \pm 1492$ & $-3576 \pm 1109$ & $-3481 \pm 389$ \\
\hline
\end{tabular}

LVEDP, left ventricular end-diastolic pressure; LVESP, left ventricular end-systolic pressure; Max + dp/dt, maximal rate of pressure development; Max $-\mathrm{dp} / \mathrm{dt}$, maximal rate of pressure decay.

${ }^{*} P<0.05$ vs. sham group.

$* * P<0.05$ vs. AAC group, $n=8$. 
A
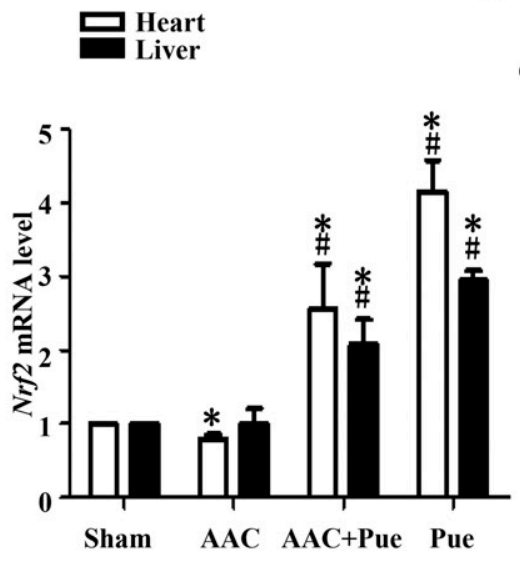

D

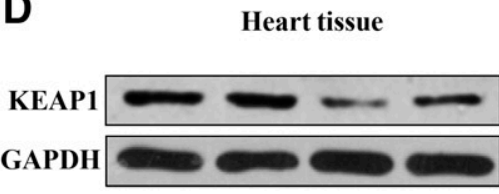

$\mathbf{E}$
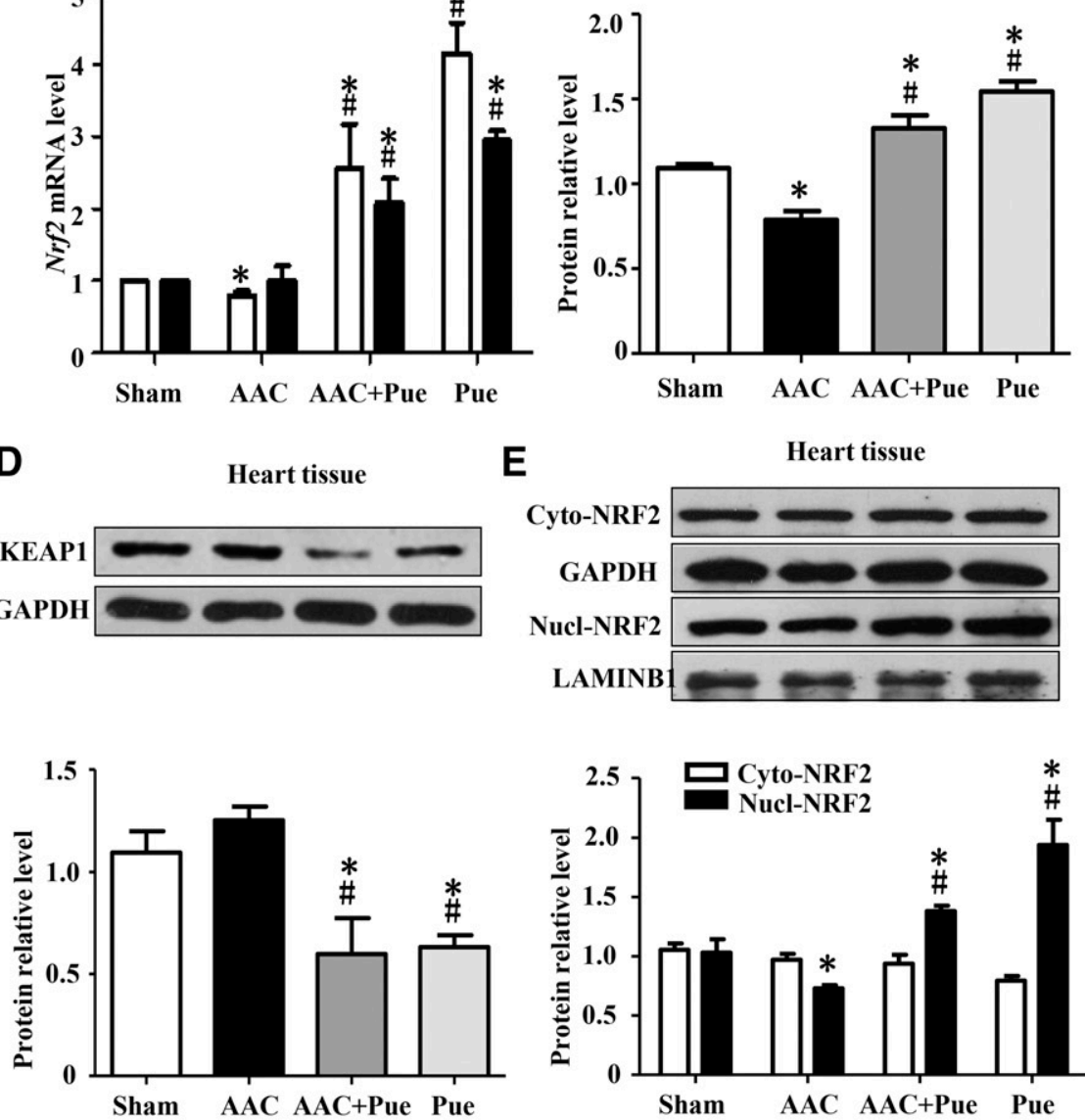
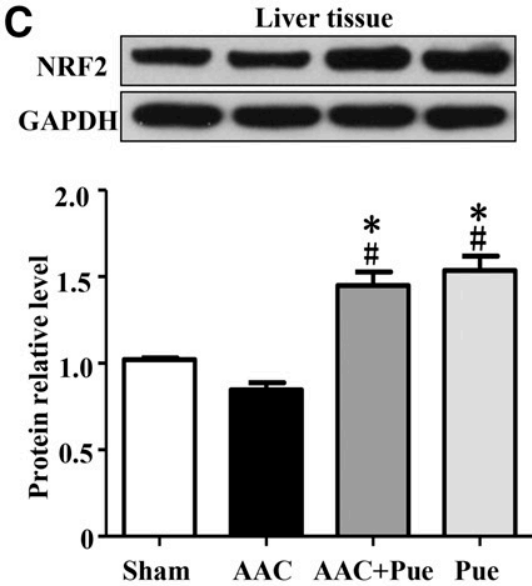

$\mathbf{F}$
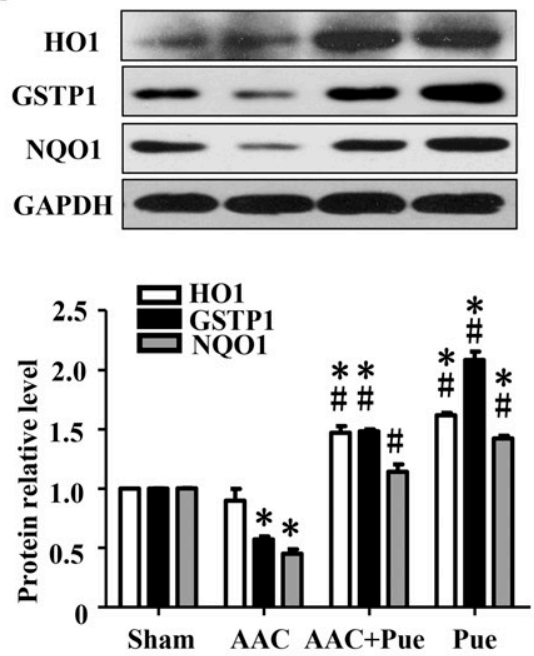

H
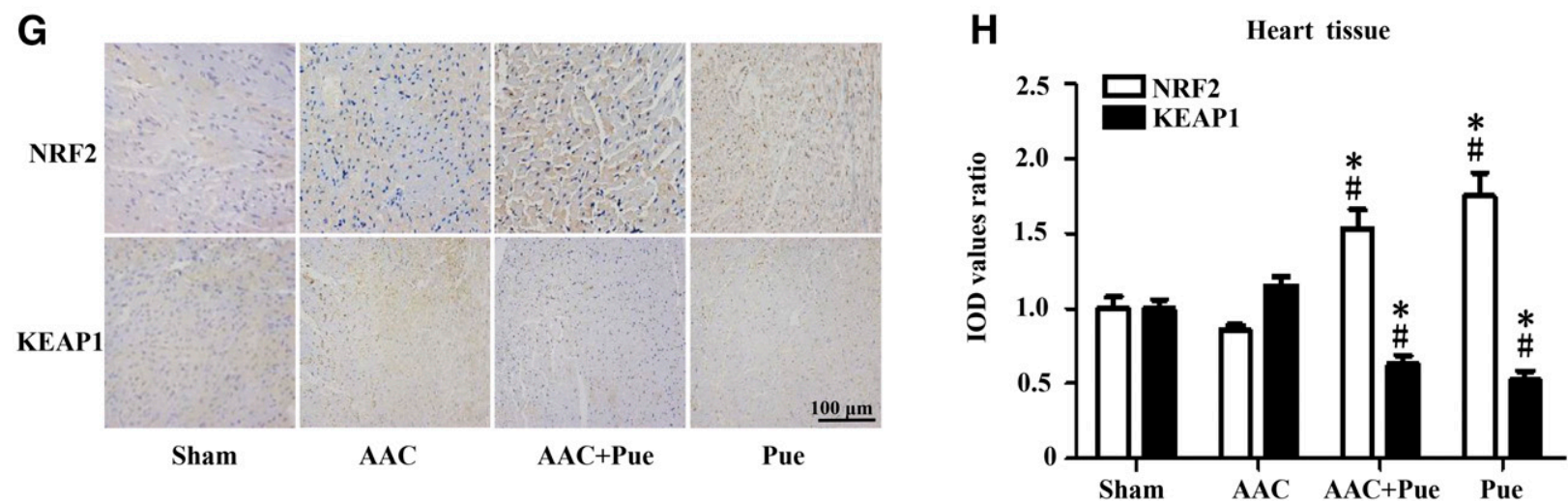

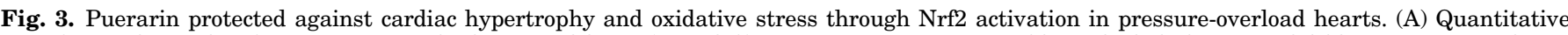

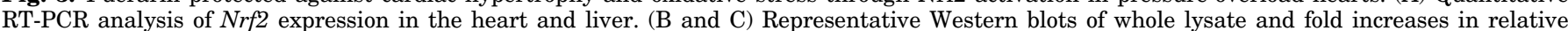

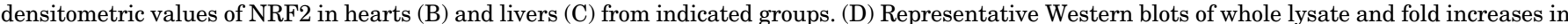

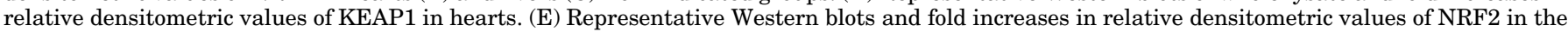

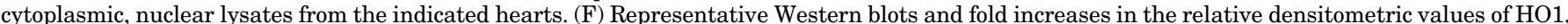

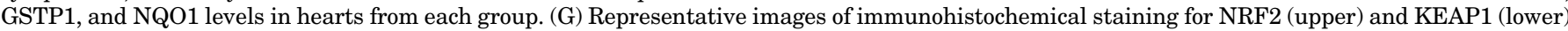

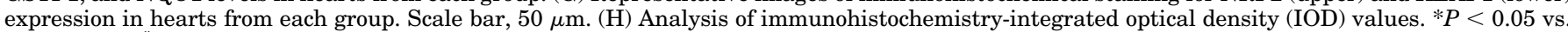
sham group; ${ }^{\#} P<0.05$ vs. AAC group, $n=8$ for each group. Each group included four male and four female rats.

were observed in Ang II-treated cardiomyocytes, which were reversed by puerarin treatment. Moreover, Ang II significantly decreased the nuclear accumulation of NRF2. Coincubation with puerarin restored the NRF2 activity induced by Ang II (Fig. 4E). Immunofluorescence staining also revealed that NRF2 accumulated in the nuclei of cardiomyocytes treated with Ang II and puerarin or puerarin alone (Fig. 4F). 
A

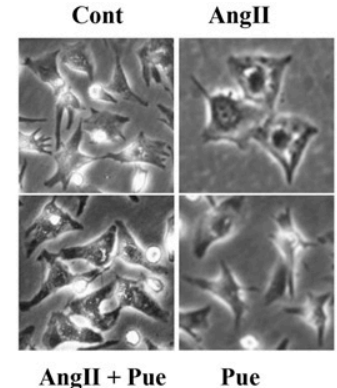

B

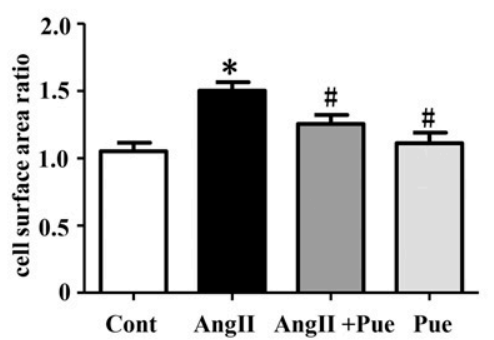

C

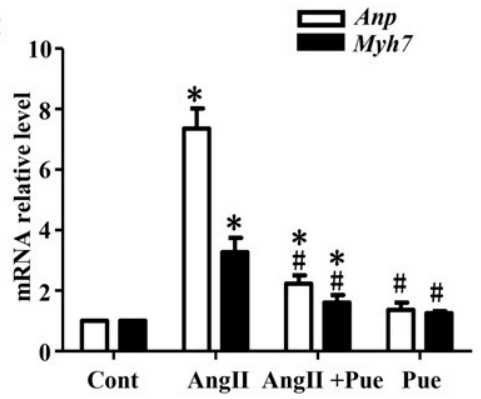

D
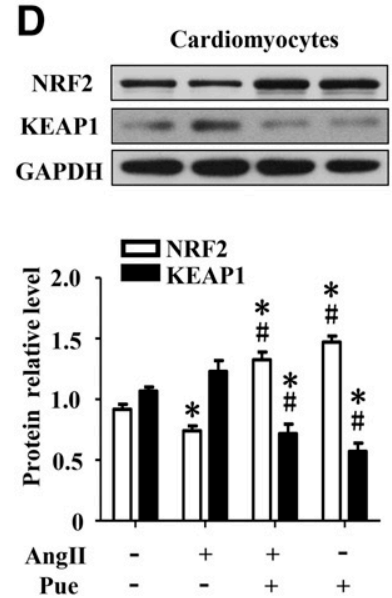

E
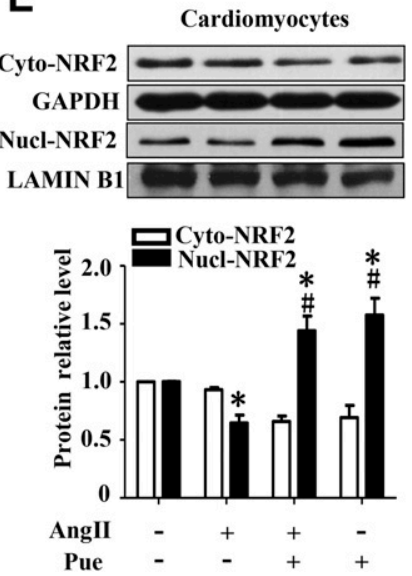

F

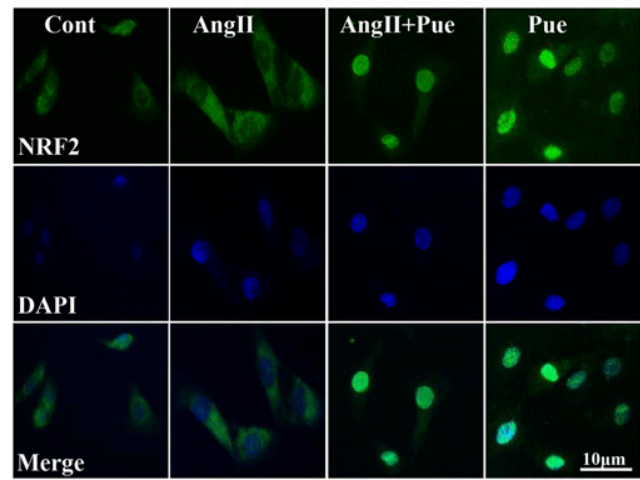

Fig. 4. Puerarin stimulated Nrf2 nucleus translocation in Ang II-induced NRCM hypertrophy. (A and B) Representative micrographs (A) and measurement of surface area (B) for cardiomyocytes subjected to different treatments as indicated. (C) RT-PCR analysis for atrial natriuretic peptide $(A n p)$ and $\beta$-myosin heavy chain (Myh7) in NRCMs subjected to indicate treatments. (D) Representative Western blots of whole lysate and fold increases in relative densitometric values of NRF2 and KEAP1 in each group. (E) Representative Western blots and fold increases in relative densitometric values of NRF2 in the cytoplasmic, nuclear lysates from the indicated groups. (F) Representative immunofluorescence images demonstrating nuclear localization of NRF2 in cardiomyocytes. ${ }^{*} P<0.05$ vs. control group; ${ }^{\#} P<0.05$ vs. Ang II group, $n=6$ for each group.

Puerarin Protected against Ang II-Induced Cardiomyocyte Hypertrophy and Oxidative Stress through the Activation of Nrf2. The role of Nrf2 in puerarin's antihypertrophy property was further confirmed via siRNA. After transfection with Nrf2 siRNA or negative control siRNA for 48 hours, cardiomyocytes were subsequently treated with Ang II ( $1 \mu \mathrm{M})$ alone, in combination with Ang II $(1 \mu \mathrm{M})$ and puerarin $(100 \mu \mathrm{M})$, or puerarin alone $(100 \mu \mathrm{M})$ for another 24 hours. Figure 5, C and D, demonstrated that using Nrf2 siRNA dramatically inhibited Nrf2 mRNA and protein expression compared with the negative control. Moreover, Nrf2 siRNA attenuated the antihypertrophic effects of puerarin. The puerarin-induced downregulation of hypertrophic indicators, such as cell-surface area and hypertrophic genes, were also partially abolished with the silencing of Nrf2 expression with specific siRNA (Fig. 5, A-C). In addition, we also evaluated the expression of HO1, GSTP1, and NQO1. Like rats in the AAC group, Ang II treatment decreased the expression of the antioxidant proteins, which was blocked by puerarin treatment (Fig. 5, D and E). The puerarin-induced antioxidative effect was abolished by silencing Nrf2 expression with specific siRNA (Fig. 5, D and E). These data further support that the puerarin-induced cardiac protection was partially mediated through transcription activator Nrf2.

Puerarin Upregulated the Expression of the Drug-Metabolizing Enzymes UGT1A1 and UGT1A9. Glucuronidation represents a major conjugative reaction catalyzed by UDP-glucuronosyltransferases (UGTs). According to our previous results, UGT1A1 and UGT1A9 significantly catalyzed the formation of puerarin metabolites, and the activity of UGT1A1 was significantly higher than that of other enzymes (Luo et al., 2012). We detected UGT1A1 and UGT1A9 expression using RT-PCR and Western blotting in the liver and heart tissues from the rats in each group. Figure 6 shows that the mRNA and protein levels of UGT1A1 and UGT1A9 were significantly increased in the livers and hearts from rats of AAC-Pue and Pue groups compared with their controls. Expression of these two metabolic enzymes was not significantly different in the AAC rats compared with the sham group (Fig. 6, A-F). Immunohistochemical staining also indicated that UGT1A1 and UGT1A9 increased in the puerarin administration groups (Fig. 6, G and H).

Puerarin Upregulated UGT1A1 and UGT1A9 Levels through Activation of Nrf2 in Cardiomyocytes. Most reports have indicated that Nrf2 may be an important regulator of the Ugt1as gene (Kundu et al., 2011); thus, we further identified the role of Nrf2 in puerarin-stimulated UGT1A1 and UGT1A9 upregulation with Nrf2 siRNA in Ang II-treated cardiomyocytes. Although the mRNA and protein levels of UGT1A1 and UGT1A9 in Ang II-treated cardiomyocytes were not significantly different, they were significantly induced after incubation with puerarin for 24 hours compared with the control cells. Moreover, after the specific downregulation of Nrf2 with siRNA, the puerarin-induced upregulation of UGT1A1 and UGT1A9 was partially abolished (Fig. 7). These data suggested that Nrf2 activation played a pivotal role in the puerarin-induced expressions of UGT1A1 and UGT1A9. 
A

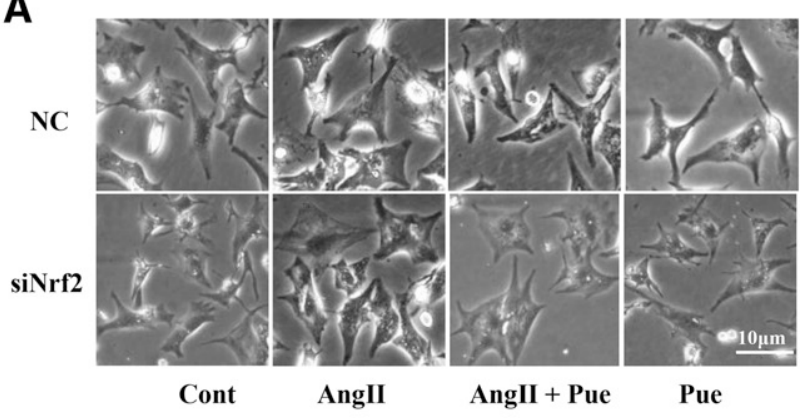

C

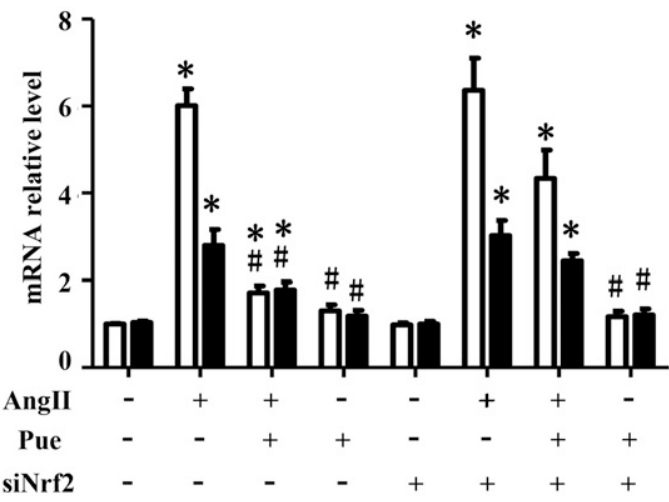

B

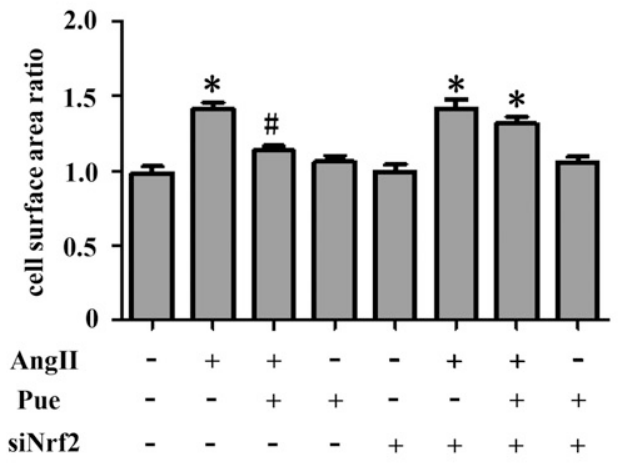

E

Cardiomyocytes
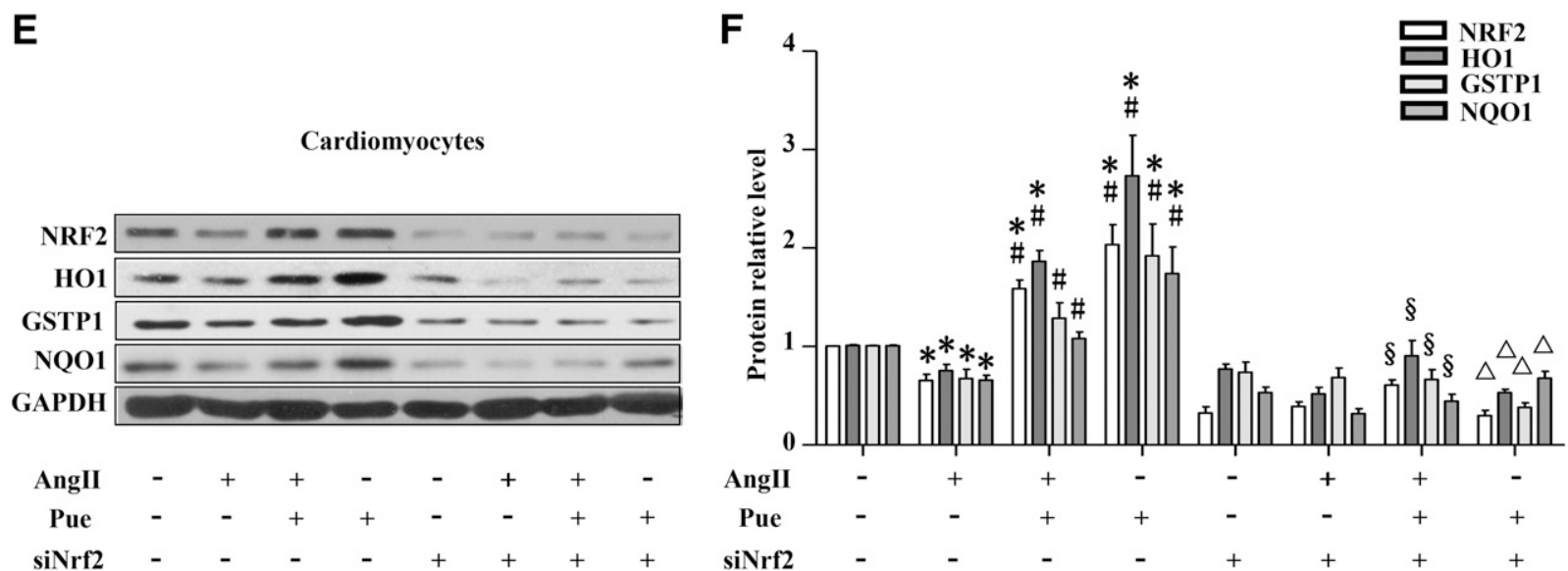

Fig. 5. Puerarin protected against Ang II-induced cardiomyocyte hypertrophy and oxidative stress through activation of Nrf2. (A and B) Representative micrographs (A) and measurement of surface area (B) for cardiomyocytes subjected to different treatments as indicated. (C and D) RT-PCR analysis for Anp, Myh7 (C), and Nrf2 (D) in NRCMs subjected to indicated treatments. (E and F) Representative Western blots of whole lysate (E) and fold changes $(F)$ in relative densitometric values of NRF2, HO1, GSTP1, and NQO1 in cultured rat ventricular cardiomyocytes under different treatments. $* P<0.05$ vs. control + negative control (NC) siRNA group. ${ }^{\sharp} P<0.05$ vs. Ang II + NC siRNA group, ${ }^{\S} P<0.05$ vs. Ang II + puerarin + NC siRNA group, ${ }^{\triangle} P<0.05$ vs. puerarin $+\mathrm{NC}$ siRNA group, $n=6$ for each group.

Puerarin-Induced Nrf2 Upregulation Is Relative to Elevated UGT1A1 and UGT1A9 Promoter Activation. To demonstrate that Nrf2 may induce the transcription of UGT1A1 or UGT1A9 by binding to the promoters of the genes, we performed ChIP-qPCR in control and puerarin-treated cardiomyocytes. Each immunoprecipitant was immunoprecipitated with anti-Nrf2 antibody. We found that puerarin significantly enhanced the binding of Nrf2 to the Ugt1a1 or Ugt1a9 promoter region observed in puerarin-treated cardiomyocytes compared with control cells and negative controls (Fig. 8, D and E).

\section{Discussion}

We and others have reported that puerarin can prevent cardiac hypertrophy induced by pressure overload (Chen et al., 2014; Yuan et al., 2014; Gang et al., 2015; Liu et al., 2015; 


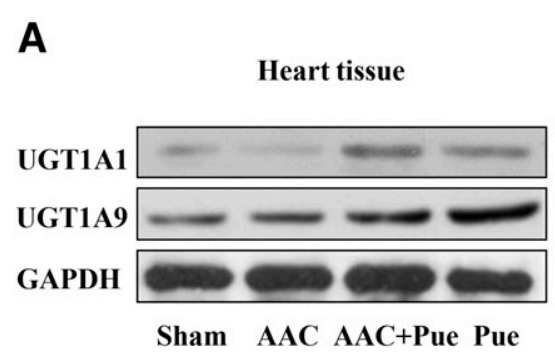

D

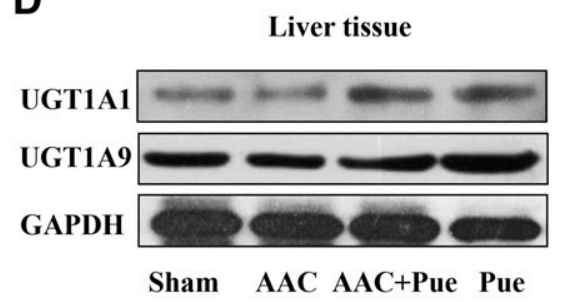

G

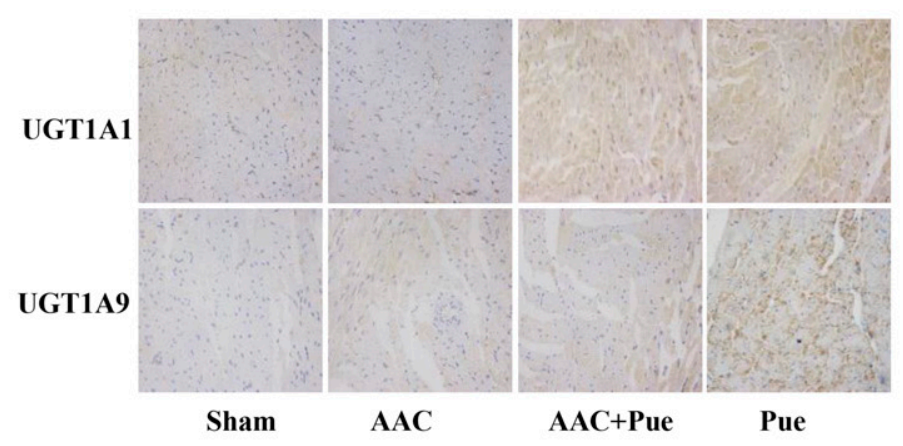

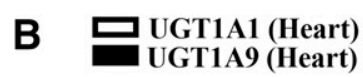

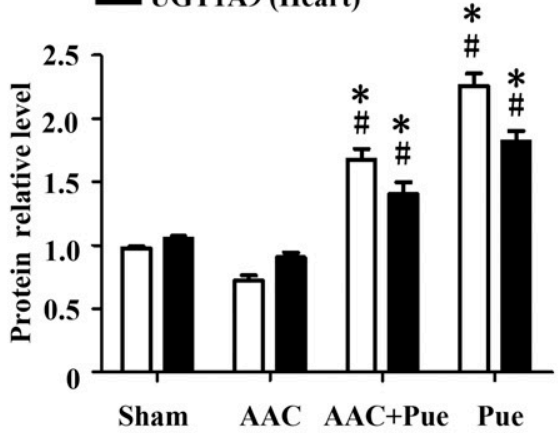

$\mathbf{E}$

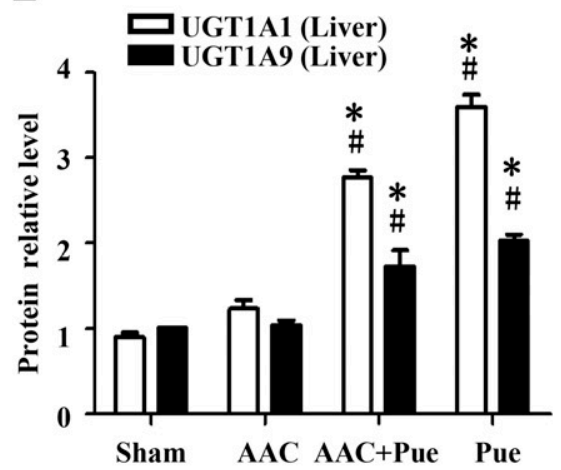

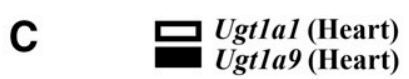

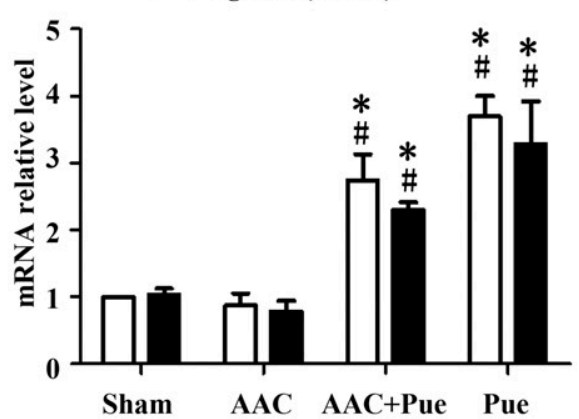

$\mathbf{F}$

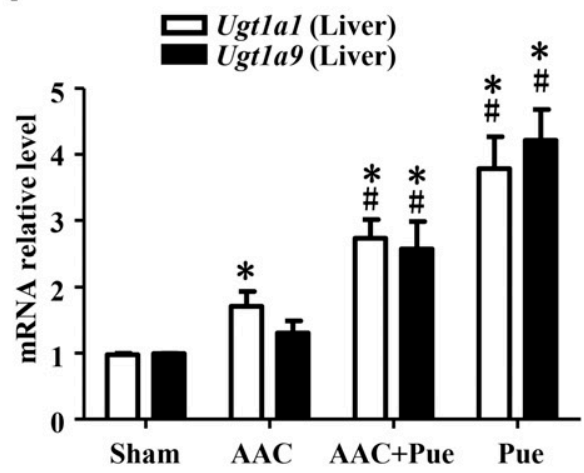

Fig. 6. Puerarin upregulated expression of drug-metabolizing enzymes UGT1A1 and UGT1A9. (A and B) Representative Western blots of UGT1A1 and UGT1A9 in whole lysates and fold changes in relative densitometric values in rat hearts from each group. (C) Fold changes in Ugt1a1 and 1a9 mRNA levels by real-time RT-PCR in rat hearts from each group. (D and E) Representative Western blots of UGT1A1 and UGT1A9 in whole lysates and fold changes in relative densitometric values in rat livers from each group. (F) Fold changes in Ugt1a1 and 1a9 mRNA levels by real-time RT-PCR in rat livers from each group. $n=8$ for each group (four male and four female rats). (G) Representative images of immunohistochemical staining from three rat hearts in each group for UGT1A1 (upper) and 1A9 (lower). (H) Quantitative integrated optical density (IOD) values of UGT1A1 and 1A9. *P $<0.05$ vs. sham group; ${ }^{\#} P<0.05$ vs. AAC group.

Zhang et al., 2016). Similarly, the present study indicated that puerarin could inhibit AAC-induced cardiac hypertrophy in rats and Ang II-induced cardiomyocytes. These results suggest that the benefits of puerarin for the cardiovascular system may depend, at least partially, on its attenuation of cardiac hypertrophy and ventricular remodeling.

Nrf2 is a member of the cap-n-collar family of transcription factors. Keap1 is a specific regulator of Nrf2 (Kensler et al., 2007). Accumulation of Nrf2 in the nucleus leads to its binding to ARE in the upstream promoter region and promotes the transcription of a battery of antioxidative genes, including Ho1, Nqo1, Gstp1, and others (Kensler et al., 2007; Zhou et al., 2014a;
Taguchi et al., 2016). It has recently been reported that pharmacologic activation of Nrf2 can reverse liver fibrosis in a mouse model of nonalcoholic steatohepatitis (Sharma et al., 2017). The Nrf2-mediated pathway has been reported to play an important role in cardiac hypertrophy and remodeling (Zhou et al., 2014a). Nrf2 knockout mice undergo cardiac hypertrophy and progress to heart failure (Erkens et al., 2015; Strom and Chen, 2017). The activation of Nrf2 regulates redox homeostasis (Jung and Kwak, 2010) and suppresses inflammation (Qu et al., 2015) and endoplasmic reticulum stress (Cominacini et al., 2015), which are involved in the pathogenesis of cardiac hypertrophy (Cominacini et al., 2015; 

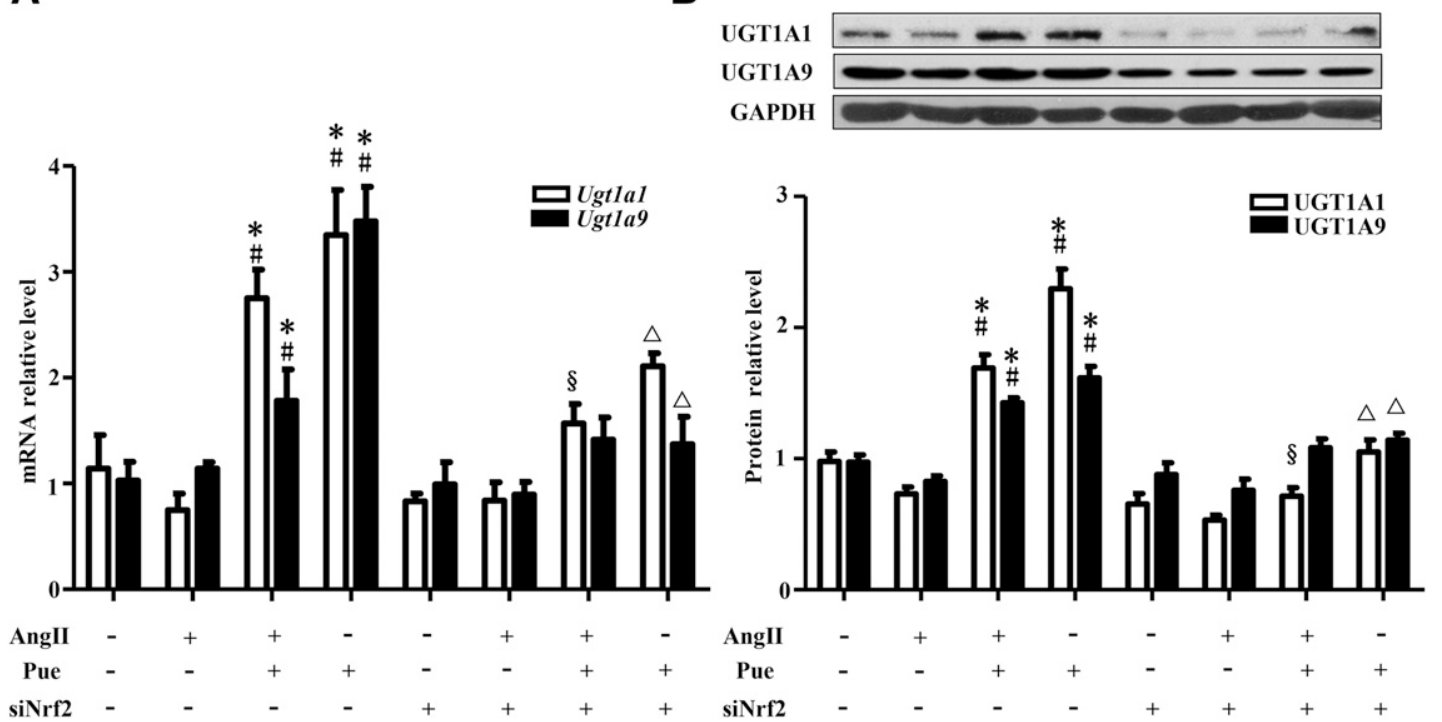

Fig. 7. Puerarin upregulated UGT1A1 and UGT1A9 levels through the activation of Nrf2 in NRCMs. (A) Fold changes in Nrf2, Ugt1a1, and 1a9 mRNA levels by real-time RT-PCR in Nrf2 siRNA-transfected NRCMs combined with indicated treatments. (B) Representative Western blots of NRF2, UGT1A1, and UGT1A9 in whole lysates and fold changes in relative densitometric values in NRCMs subjected to the indicated treatments. $* P<0.05$ vs. control + negative control (NC) siRNA group; ${ }^{\#} P<0.05 \mathrm{vs}$. Ang II + negative control (NC) siRNA group, ${ }^{8} P<0.05 \mathrm{vs}$. Ang II + puerarin + negative control (NC) siRNA group, ${ }^{\triangle} P<0.05$ vs. puerarin + NC siRNA group, $n=6$ for each group.

Tham et al., 2015). Accumulating data have suggested that oxidative stress is an important mechanism in the development of cardiac hypertrophy (Tham et al., 2015). We showed that puerarin could protect against cardiomyocyte hypertrophy induced by Ang II via inhibiting oxidative stress in NRCMs (Hou et al., 2017). These results were further confirmed by current findings that puerarin significantly increased the expression of Nrf2 in the hearts of pressure-overload rats and NRCMs treated by Ang II. Puerarin treatment also decreased the protein level of Keap1 in the hearts of pressureoverload rats and NRCMs treated by Ang II. Additionally, the level of Nrf2 protein in the nucleus was significantly increased in both hearts and NRCMs, although the level of Nrf2 protein in cytosol remained similar. The upregulation of nuclear Nrf2 enhanced the expression of HO1, NQO1, and GSTP1 in the myocardium of pressure-overload rats and NRCMs treated with Ang II. Downregulation of Nrf2 by siNrf2 could inhibit the increase of these antioxidative genes induced by puerarin in NRCMs treated with Ang II, and the antihypertrophic efficacy of puerarin was also simultaneously suppressed. These results indicated that puerarin could prevent cardiac hypertrophy via Nrf2-mediated antioxidative mechanisms.

Besides Keap1, Nrf2 is negatively regulated by another ubiquitin ligase substrate adaptor, $\beta$-transducin repeatcontaining protein $(\beta$-TrCP). Keap1 and $\beta$-TrCP respectively allow cullin-3 and cullin-1 to ubiquitylate Nrf2. In the case of Keap1, thiol-reactive electrophiles cause inactivation of its adaptor function by modifying Cys residues, and this blunts ubiquitylation of Nrf2. In the case of $\beta$-TrCP, it recognizes a DSGIS-containing phosphodegron in Nrf2 that is formed by glycogen synthase kinase-3 (GSK-3). Thus, when Nrf2 is phosphorylated by GSK-3, the binding of $\beta$-TrCP to Nrf2 then enables cullin-1 to ubiquitylate Nrf2, which leads to proteasomal degradation of the transcription factor (Rada et al., 2011; Chowdhry et al., 2013). Using Keap1-null MEFs, it has been shown that a variety of phytochemical inducers can activate
Nrf2 in a Keap1-independent manner, presumably by inactivating GSK-3 directly, increasing the inhibitory phosphorylation of Ser-9/21 in GSK-3, or by preventing it from phosphorylating Nrf2 (Hayes et al., 2016). It has been reported previously that puerarin exerts hepatoprotective effects via downregulating GSK-3 $\beta$ (Katsiki et al., 2013). It therefore seems likely that puerarin may inactivate GSK-3 or inhibit a priming kinase.

Transcription factors, including AhR, CAR, and PXR, are important regulators for the expression of drug-metabolizing enzymes. Intriguingly, feedback loops are generated when activators of the transcription factor are substrates of drugmetabolizing enzymes, which are regulated by the transcription factors; this has been proposed as an important mechanism for maintaining homeostasis in drug-metabolizing enzymes (Bock, 2012, 2014). Previous evidence suggests that Nrf2 may be a key transcription factor regulating the expression of genes for xenobiotic metabolism (Kensler et al., 2007). Nrf2 has been reported to upregulate the expression of UGT1A1 and UGT1A6 (Thimmulappa et al., 2002; Kundu et al., 2011; Bai et al., 2016). In our study, UGT1A1 and UGT1A9 mRNA and protein were detected in the livers and hearts of rats, as well as in NRCMs. In liver and heart tissues of rats after 7 weeks of AAC, puerarin significantly increased the mRNA and protein levels of UGT1A1 and UGT1A9. In NRCMs treated by Ang II, the mRNA and protein levels of UGT1A1 and UGT1A9 were also upregulated by puerarin. The downregulation of Nrf2 by siNrf2 inhibited the upregulation of UGT1A1 and 1A9 protein levels induced by puerarin in Ang II-treated NRCMs. Taken together, these results suggest that puerarin may induce its metabolic enzymes UGT1A1 and 1A9 expression via Nrf2. ChIP assay results further confirmed that puerarin enhanced the binding of Nrf2 to the UGT1A1 and 1A9 promoter in NRCMs.

This autoregulatory circuit between puerarin and its metabolic enzymes may be similar to the mechanisms underlying the metabolism of other biologic compounds. In the early 
A
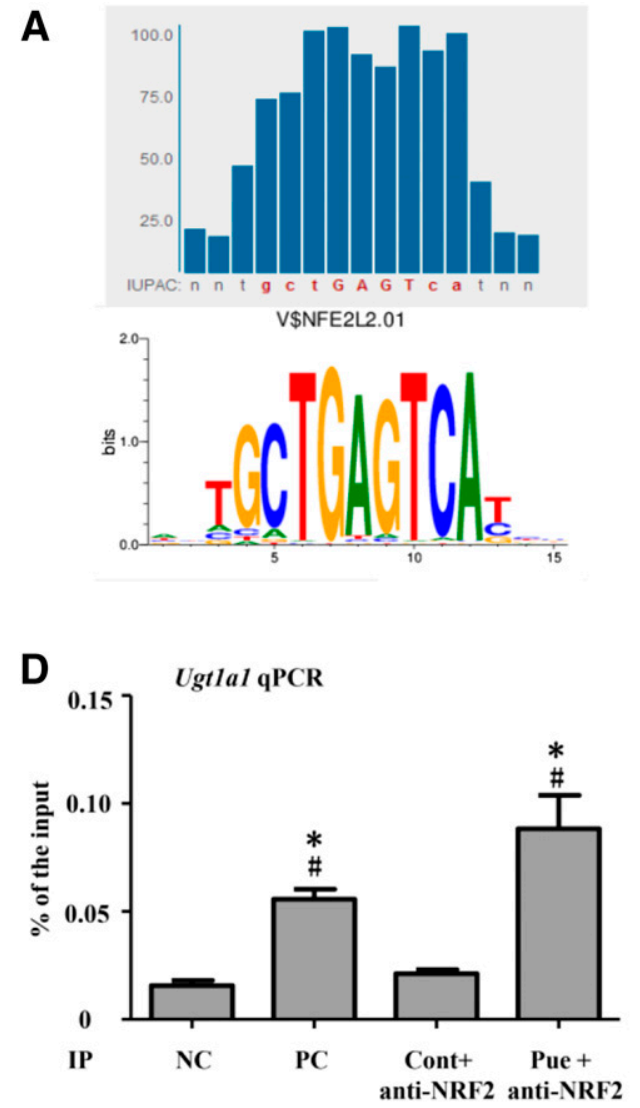

B

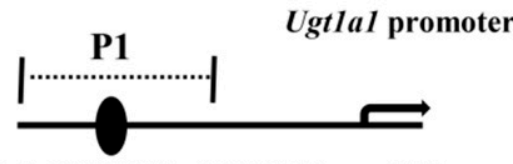

>chr9:95294695+95294810 116bp

ttgacagctgagtcctggaaagg

C

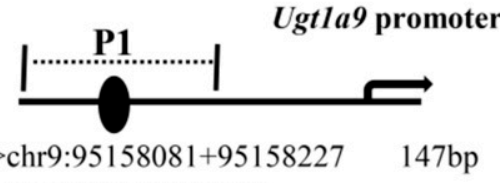

aaaaaagctgagtaagactccat

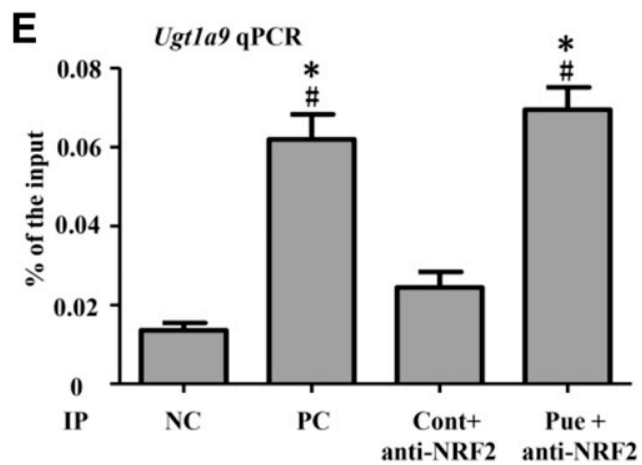

Fig. 8. Puerarin enhanced Nrf2 binding to UGT1A1 and UGT1A9 promoter regions. (A) Nucleotides marked red show a high information content, which in capital letters denotes the core sequence used by Matlnspector software. Genomic binding sites were identified by ChIP-Seq. (B and C) Sequences of Ugt1a1 and Ugt1a9 promoters. (D and E) Chromatin was immunoprecipitated with anti-NRF2 antibody, positive control anti-RNA polymerase II, and negative control normal mouse IgG. Nrf2 binding to Ugt1a1 or $1 a 9$ promoter was determined by qPCR with Ugt1a1 or $1 a 9$ specific primers. The same primers were also used to amplify Ugt1a1 or $1 a 9$ promoter regions to show $1 \%$ input DNA. Graphic representation of the results obtained in ChIP-qPCR with the "percent input" method. $* P<0.05$ vs. NC group, ${ }^{\#} P<0.05$ vs. Cont + antiNRF2 group, $n=6$ for each group. 1980s, Boutin et al. (1983) reported that xenobiotics, such as eugenol and 4-methylumbelliferone, induce UGT activity and enhance their own conjugation. Flavone compounds have been reported to increase the expression and activity of UGT1A1 in some cell lines (Galijatovic et al., 2000; Walle et al., 2000; Kundu et al., 2011). Similarly, bilirubin is an activator of AhR (Togawa et al., 2008) and CAR (Huang et al., 2003). Bilirubin subsequently induces the expression of UGT1A1 and enhances the clearance of bilirubin, which indicates that the feedback loops between bilirubin and AhR/CAR-induced UGT1A1 are useful to maintain the homeostasis of bilirubin. Similar autoregulatory circuits were also demonstrated between quercetin and AhR/Nrf2-regulated UGT1A1 (Bock, 2012). Our results suggested that there may be an autoregulatory circuit between puerarin and Nrf2-regulated UGT1A1/1A9.

It is important to maintain the homeostasis of metabolizing enzymes of puerarin because intravenous administration of puerarin has been associated with severe side effects, such as intravascular hemolysis. The mechanism for the development of intravascular hemolysis remains uncertain, which may be associated with changes in membrane lipids and the composition of erythrocyte membrane proteins after incubation with puerarin in vitro, perhaps in a concentration-dependent manner (Hou et al., 2011). After puerarin administration, upregulation of UGT1A1 and UGT1A9 will enhance puerarin metabolism. From our point of view, this will have at least two effects. First, the concentration of puerarin in the plasma will decrease, which may be an initial protective effect for the incidence of severe side effects, such as intravascular hemolysis. Second, the metabolites of puerarin will increase, including puerarin-7-O-glucuronide. Our previous study suggested that puerarin-7-O-glucuronide can exert antioxidative and antihypertrophic efficacy in cardiomyocytes (Hou et al., 2017), which indicates that the upregulation of UGT1A1 and UGT1A9 induced by puerarin may decrease the risk of severe side effects without significantly changing the antihypertrophic effects as compensated by the antihypertrophic efficacy of its metabolites. Interestingly, Nrf2 appears to be an important transcription factor not only underlying the preventative efficacy of puerarin against cardiac hypertrophy but also in upregulating the metabolizing enzymes of puerarin.

In conclusion, puerarin can enhance the expression of transcription factor Nrf2 and its accumulation in the nucleus. On the one hand, the upregulation and nuclear accumulation of Nrf2 can promote the expression of antioxidative genes and prevent cardiac hypertrophy. On the other hand, the upregulation and nuclear accumulation of $\mathrm{Nrf2}$ can increase the expression of metabolizing enzymes of puerarin, including UGT1A1 and UGT1A9. These dual effects of transcription factor Nrf2 and potential autoregulatory circuits between puerarin and Nrf2-regulated UGT1A1/1A9 may be important to maintain antihypertrophic efficacy and to limit the risk of the potential side effects of puerarin.

\section{Authorship Contributions}

Participated in research design: Zhao, Hou, Cai, X.W. Liu, Luo, M.-S. Chen.

Conducted experiments: Zhao, Hou, Cai, X.W. Liu, A.-Q. Li, Luo, Huang, L.-R. Li.

Contributed new reagents or analytic tools: Ou, Mai, Xiong. Performed data analysis: Zhao, Hou, Cai, X.W. Liu.

Wrote or contributed to the writing of the manuscript: Zhao, Hou, Cai, X.W. Liu, Luo. 


\section{References}

Bai X, Chen Y, Hou X, Huang M, and Jin J (2016) Emerging role of NRF2 in chemoresistance by regulating drug-metabolizing enzymes and efflux transporters. Drug Metab Rev 48:541-567.

Bock KW (2012) Human UDP-glucuronosyltransfeases: feedback loops between substrates and ligands of their transcription factors. Biochem Pharmacol 84: $1000-1006$

Bock KW (2014) Homeostatic control of xeno- and endobiotics in the drugmetabolizing enzyme system. Biochem Pharmacol 90:1-6.

Boutin JA, Batt AM, and Siest G (1983) Effect of pretreatment with hydroxylated xenobiotics on the activities of rat liver UDP-glucuronosyl-transferases. Xenobiotica 13 755-761.

Chen G, Pan S-Q, Shen C, Pan S-F, Zhang X-M, and He Q-Y (2014) Puerarin inhibits angiotensin II-induced cardiac hypertrophy via the redox-sensitive ERK1/2, p38 and NF-кB pathways. Acta Pharmacol Sin 35:463-475.

Chowdhry S, Zhang Y, McMahon M, Sutherland C, Cuadrado A, and Hayes JD (2013) Nrf2 is controlled by two distinct $\beta$-TrCP recognition motifs in its Neh6 domain, one of which can be modulated by GSK-3 activity. Oncogene 32:3765-3781.

Cominacini L, Mozzini C, Garbin U, Pasini A, Stranieri C, Solani E, Vallerio P, Tinelli IA, and Fratta Pasini A (2015) Endoplasmic reticulum stress and Nrf2 signaling in cardiovascular diseases. Free Radic Biol Med 88 (Pt B):233-242.

Erkens R, Kramer CM, Lückstädt W, Panknin C, Krause L, Weidenbach M, Dirzka J, Krenz T, Mergia E, Suvorava T, et al. (2015) Left ventricular diastolic dysfunction in Nrf2 knock out mice is associated with cardiac hypertrophy, decreased expression of SERCA2a, and preserved endothelial function. Free Radic Biol Med $\mathbf{8 9}$ 906-917.

Galijatovic A, Walle UK, and Walle T (2000) Induction of UDP-glucuronosyltransferase by the flavonoids chrysin and quercetin in Caco-2 cells. Pharm Res 17:21-26.

Gang C, Qiang C, Xiangli C, Shifen P, Chong S, and Lihong L (2015) Puerarin suppresses angiotensin II-induced cardiac hypertrophy by inhibiting NADPH oxidase activation and oxidative stress-triggered AP-1 signaling pathways. J Pharm Pharm Sci 18:235-248.

Gao L, Ji X, Song J, Liu P, Yan F, Gong W, Dang S, and Luo Y (2009) Puerarin protects against ischemic brain injury in a rat model of transient focal ischemia. Neurol Res 31:402-406.

Hayes JD, Ebisine K, Sharma RS, Chowdhry S, Dinkova-Kostova AT, and Sutherland C (2016) Regulation of the CNC-bZIP transcription factor Nrf2 by Keap1 and the axis between GSK-3 and $\beta$-TrCP. Curr Opin Toxicol 1:92-103.

Hou N, Cai B, Ou CW, Zhang ZH, Liu XW, Yuan M, Zhao GJ, Liu SM, Xiong LG, Luo JD, et al. (2017) Puerarin-7-O-glucuronide, a water-soluble puerarin metabolite, prevents angiotensin II-induced cardiomyocyte hypertrophy by reducing oxidative stress. Naunyn Schmiedebergs Arch Pharmacol 390:535-545.

Hou SZ, Su ZR, Chen SX, Ye MR, Huang S, Liu L, Zhou H, and Lai XP (2011) Role of the interaction between puerarin and the erythrocyte membrane in puerarininduced hemolysis. Chem Biol Interact 192:184-192.

Hsu FL, Liu IM, Kuo DH, Chen WC, Su HC, and Cheng JT (2003) Antihyperglycemic effect of puerarin in streptozotocin-induced diabetic rats. $J$ Nat Prod 66:788-792.

Huang J, Tang X, Liang X, Wen Q, Zhang S, Xuan F, Jian J, Lin X, and Huang R (2015) The effects of 17-methoxyl-7-hydroxy-benzene-furanchalcone on pressure overload-induced cardiac remodeling in rats and the endothelial mechanisms based on PGI2. Cell Physiol Biochem 36:1004-1014.

Huang W, Zhang J, Chua SS, Qatanani M, Han Y, Granata R, and Moore DD (2003) Induction of bilirubin clearance by the constitutive androstane receptor (CAR) Proc Natl Acad Sci USA 100:4156-4161.

Jung KA and Kwak MK (2010) The Nrf2 system as a potential target for the development of indirect antioxidants. Molecules 15:7266-7291.

Kamo T, Akazawa H, and Komuro I (2015) Cardiac nonmyocytes in the hub of cardiac hypertrophy. Circ Res 117:89-98.

Katsiki N, Karagiannis A, and Mikhailidis DP (2013) Diabetes, bilirubin and amputations: is there a link? Diabetologia 56:683-685

Kensler TW, Wakabayashi N, and Biswal S (2007) Cell survival responses to environmental stresses via the Keap1-Nrf2-ARE pathway. Annu Rev Pharmacol Toxicol 47: $89-116$.

Ku HC, Lee SY, Wu YA, Yang KC, and Su MJ (2016) A model of cardiac remodeling through constriction of the abdominal aorta in rats. J Vis Exp (118): e54818.

Kundu R, Dasgupta S, Biswas A, Bhattacharya S, Pal BC, Bhattacharya S, Rao PG Barua NC, Bordoloi M, and Bhattacharya S (2011) Carlinoside reduces hepatic bilirubin accumulation by stimulating bilirubin-UGT activity through Nrf2 gene expression. Biochem Pharmacol 82:1186-1197.

Li H, Dong L, Liu Y, Wang G, Wang G, and Qiao Y (2014) Biopharmaceutics classification of puerarin and comparison of perfusion approaches in rats. Int J Pharm 466:133-138.

Liu B, Wu Z, Li Y, Ou C, Huang Z, Zhang J, Liu P, Luo C, and Chen M (2015) Puerarin prevents cardiac hypertrophy induced by pressure overload through activation of autophagy. Biochem Biophys Res Commun 464:908-915.

Luo CF, Cai B, Hou N, Yuan M, Liu SM, Ji H, Xiong LG, Xiong W, Luo JD, and Chen MS (2012) UDP-glucuronosyltransferase 1A1 is the principal enzyme responsible for puerarin metabolism in human liver microsomes. Arch Toxicol 86:1681-1690.

Luo CF, Hou N, Tian J, Yuan M, Liu SM, Xiong LG, Luo JD, and Chen MS (2013) Metabolic profile of puerarin in rats after intragastric administration of puerarin solid lipid nanoparticles. Int $J$ Nanomedicine 8:933-940.

Luo CF, Yuan M, Chen MS, Liu SM, Huang BY, Liu XW, and Zhu L (2011a) Determination of puerarin in rat plasma by rapid resolution liquid chromatography tandem mass spectrometry in positive ionization mode. $J$ Chromatogr B Analyt Technol Biomed Life Sci 879:1497-1501.

Luo CF, Yuan M, Chen MS, Liu SM, Zhu L, Huang BY, Liu XW, and Xiong W (2011b) Pharmacokinetics, tissue distribution and relative bioavailability of puerarin solid lipid nanoparticles following oral administration. Int J Pharm 410:138-144.

Mackenzie PI, Hu DG, and Gardner-Stephen DA (2010) The regulation of UDPglucuronosyltransferase genes by tissue-specific and ligand-activated transcription factors. Drug Metab Rev 42:99-109.

Oka T, Akazawa H, Naito AT, and Komuro I (2014) Angiogenesis and cardiac hypertrophy: maintenance of cardiac function and causative roles in heart failure. Circ Res 114:565-571.

Qu C, Li B, Lai Y, Li H, Windust A, Hofseth LJ, Nagarkatti M, Nagarkatti P, Wang XL, Tang D, et al. (2015) Identifying panaxynol, a natural activator of nuclear factor erythroid-2 related factor 2 (Nrf2) from American ginseng as a suppressor of inflamed macrophage-induced cardiomyocyte hypertrophy. J Ethnopharmacol 168: 326-336.

Rada P, Rojo AI, Chowdhry S, McMahon M, Hayes JD, and Cuadrado A (2011) $\mathrm{SCF} / \beta$-TrCP promotes glycogen synthase kinase 3-dependent degradation of the Nrf2 transcription factor in a Keap1-independent manner. Mol Cell Biol $\mathbf{3 1}$ $1121-1133$

Reddy DS, Singh M, Ghosh S, and Ganguly NK (1996) Role of cardiac reninangiotensin system in the development of pressure-overload left ventricular hypertrophy in rats with abdominal aortic constriction. Mol Cell Biochem 155:1-11.

Sharma RS, Harrison DJ, Kisielewski D, Cassidy DM, McNeilly AD, Gallagher JR, Walsh SV, Honda T, McCrimmon RJ, Dinkova-Kostova AT, et al. (2017) Experimental nonalcoholic steatohepatitis and liver fibrosis are ameliorated by pharmacologic activation of Nrf2 (NF-E2 p45-related factor 2). Cell Mol Gastroenterol Hepatol 5:367-398.

Simpson P and Savion S (1982) Differentiation of rat myocytes in single cell cultures with and without proliferating nonmyocardial cells: cross-striations, ultrastructure, and chronotropic response to isoproterenol. Circ Res 50:101-116.

Song XP, Chen PP, and Chai XS (1988) [Effects of puerarin on blood pressure and plasma renin activity in spontaneously hypertensive rats]. Zhongguo Yao Li Xue Bao 9:55-58.

Strom J and Chen QM (2017) Loss of Nrf2 promotes rapid progression to heart failure following myocardial infarction. Toxicol Appl Pharmacol 327:52-58.

Sugatani J, Nishitani S, Yamakawa K, Yoshinari K, Sueyoshi T, Negishi M, and Miwa M (2005) Transcriptional regulation of human UGT1A1 gene expression: activated glucocorticoid receptor enhances constitutive androstane receptor/ pregnane X receptor-mediated UDP-glucuronosyltransferase 1A1 regulation with glucocorticoid receptor-interacting protein 1. Mol Pharmacol 67:845-855.

Taguchi K, Takaku M, Egner PA, Morita M, Kaneko T, Mashimo T, Kensler TW, and Yamamoto M (2016) Generation of a new model rat: Nrf2 knockout rats are sensitive to aflatoxin B1 toxicity. Toxicol Sci 152:40-52.

Tham YK, Bernardo BC, Ooi JYY, Weeks KL, and McMullen JR (2015) Pathophysiology of cardiac hypertrophy and heart failure: signaling pathways and novel therapeutic targets. Arch Toxicol 89:1401-1438.

Thimmulappa RK, Mai KH, Srisuma S, Kensler TW, Yamamoto M, and Biswal S (2002) Identification of Nrf2-regulated genes induced by the chemopreventive agent sulforaphane by oligonucleotide microarray. Cancer Res 62:5196-5203.

Togawa H, Shinkai S, and Mizutani T (2008) Induction of human UGT1A1 by bilirubin through AhR dependent pathway. Drug Metab Lett 2:231-237.

Walle T, Otake Y, Galijatovic A, Ritter JK, and Walle UK (2000) Induction of UDPglucuronosyltransferase UGT1A1 by the flavonoid chrysin in the human hepatoma cell line hep G2. Drug Metab Dispos 28:1077-1082.

Yan LP, Chan SW, Chan ASC, Chen SL, Ma XJ, and Xu HX (2006) Puerarin decreases serum total cholesterol and enhances thoracic aorta endothelial nitric oxide synthase expression in diet-induced hypercholesterolemic rats. Life Sci $\mathbf{7 9}$ : 324-330.

Yuan Y, Zong J, Zhou H, Bian ZY, Deng W, Dai J, Gan HW, Yang Z, Li H, and Tang QZ (2014) Puerarin attenuates pressure overload-induced cardiac hypertrophy. $J$ Cardiol 63:73-81.

Zhang S, Chen S, Shen Y, Yang D, Liu X, Sun-Chi AC, and Xu H (2006) Puerarin induces angiogenesis in myocardium of rat with myocardial infarction. Biol Pharm Bull 29:945-950.

Zhang X, Liu Y, and Han Q (2016) Puerarin attenuates cardiac hypertrophy partly through increasing Mir-15b/195 expression and suppressing non-canonical transforming growth factor Beta (Tgfß) signal pathway. Med Sci Monit 22:1516-1523.

Zhou S, Sun W, Zhang Z, and Zheng Y (2014a) The role of Nrf2-mediated pathway in cardiac remodeling and heart failure. Oxid Med Cell Longev 2014:260429.

Zhou YX, Zhang H, and Peng C (2014b) Puerarin: a review of pharmacological effects. Phytother Res 28:961-975.

Address correspondence to: Cheng-Feng Luo, Department of Cardiology, The Second Affiliated Hospital of Guangzhou Medical University, Guangzhou Institute of Cardiovascular Disease, 250 Changgangdong Road, Guangzhou 510260, China. E-mail: rocenphone@gzhmu.edu.cn; or Min-Sheng Chen, Department of Cardiology, Zhujiang Hospital, Southern Medical University, Guangdong provincial Center of Biomedical Engineering for Cardiovascular Disease, No. 1023, Shatai Nan Road, Guangzhou 510280, China. E-mail: gzminsheng@vip.163.com 